\title{
DEVELOPMENT OF A TECHNOLOGY FOR PRODUCING A STABLE INJECTABLE DOSAGE FORM OF A HYDROPHOBIC INDOLOCARBAZOLE DERIVATIVE
}

\author{
MARIA DMITRIEVA ${ }^{1 *}$, ALEXANDER KOLPAKSIDI'1,2, OLGA ORLOVA ${ }^{1}$, ELENA IGNATYEVA ${ }^{1}$, ANNA LANTSOVA, \\ LUDMILA NIKOLAEVA ${ }^{1,2}$, IVAN KRASNYUK ${ }^{2}$
}

${ }^{1}$ N. N. Blokhin National Medical Research Center of Oncology, Moscow, Russia, 2Sechenov University, Moscow, Russia

*Email: dmitrieva.m@ronc.ru

Received: 08 Jul 2021, Revised and Accepted: 08 Sep 2021

\begin{abstract}
Objective: Development of a technology for the production of a stable injectable dosage form (IDF) of indolocarbazole derivative LHS-1269.

Methods: LHS-1269 is an active pharmaceutical ingredient that was synthesized in the N. N. Blokhin National Medical Research Center of Oncology of the Ministry of Health of the Russian Federation. The IDF includes dimethyl sulfoxide (DMSO), 95\% ethanol, Kollidon ${ }^{\circledR} 17$ PF and water for injection. Magnetic stirrer and overhead stirrer with a propeller stirring element were used to prepare the model solution of the IDF of LHS-1269. Sterilizing filtration of the solution was performed with $0 . z 0.22$ um polycarbonate, cellulose, polyvinylidene fluoride, poly ethersulfone and nylon membrane filters. The aqueous solution of LHS-1269 was lyophilized in Edwards Minifast D0.2 freeze dryer. Assay of LHS-1269 was performed by spectrophotometry at $320 \pm 3 \mathrm{~nm}$. Potentiometry was used to measure $\mathrm{pH}$, a viscosimetry method was used to measure the viscosity of the solutions.
\end{abstract} The average weight was estimated by weighing a sample of 10 vials with the concentrate.

Results: $0.5 \%$ aqueous solution of LHS-1269 was produced by mixing the solution of the active substance in DMSO and ethanol with an aqueous solution of polyvinylpyrrolidone gradually at the ratio of LHS-1269/DMSO/ethanol/Kollidon® of $1 / 11 / 32 / 40$ by weight. The aqueous solution of the study substance cannot be lyophilized, so a sequence of technological operations was presented to produce an anhydrous concentrate "LHS1269 , concentrate for solution for injection and infusion $25 \mathrm{mg}$ ".

Conclusion: A technology was developed to produce a stable IDF of a hydrophobic indolocarbazole derivative LHS-1269, a high-potential antitumor drug.

Keywords: Hydrophobic indolocarbazole derivative, Dimethyl sulfoxide, Solubilization, Dosage form, Freeze drying, Concentrate

(C) 2021 The Authors. Published by Innovare Academic Sciences Pvt Ltd. This is an open access article under the CC BY license (https://creativecommons.org/licenses/by/4.0/) DOI: https://dx.doi.org/10.22159/ijap.2021v13i6.42685. Journal homepage: https://innovareacademics.in/journals/index.php/ijap

\section{INTRODUCTION}

Indolocarbazoles are natural and synthetic compounds that have attracted considerable attention due to their distinctive structural features and therapeutic potential. The class of indolocarbazoles includes 5 subclasses with different structures of the planar aromatic ring. The subclasses correspond to 5 isomers of the polycycle structure: indolo[2,3- $a]$ carbazole, indolo[2,3- $b]$ carbazole, indolo[2,3-c]carbazole, indolo[3,2-a]carbazole and indolo[3,2$b]$ carbazole. The widest, most biologically significant, and wellknown is the indolo[2,3-a]carbazole subclass that includes mostly substances with a structural basis of indolo[2,3-a]pyrrolo[3,4c]carbazole ring, where 2 indole fragments are connected to amide or imide group via benzene ring. The indole fragments are connected to the carbohydrate fragment via 1 link (rebeccamycin group) or 2 links (staurosporine and its derivatives) [1].

Clinical studies of various indolocarbazole derivatives demonstrated a wide range of potential therapeutic indications and good compatibility with the known drugs. Indolo[2,3-a]carbazoles have the most pronounced antitumor effect, in line with the antibacterial, antiparasitic and other types of biological activity. The antitumor effect is based on various mechanisms of action, including intercalation in DNA, inhibition of topoisomerases I and II, protein kinases $\mathrm{C}$ and A, cyclin-dependent kinases [2, 3].

The chemical synthesis laboratory of N. N. Blokhin National Medical Research Center of Oncology of the Ministry of Health of the Russian Federation developed an original technology to produce indolo[2,3a]pyrollo[3,4-c]carbazole-5,7-dione N-glycosides (fig. 1) with a cytotoxic and antitumor activity [4]. Up to date, this method has been used to synthesize and study more than 300 indolocarbazole derivatives. A few active compounds with different carbohydrate residues, including LHS-1269, were selected as antitumor drug candidates for the next phases of development.

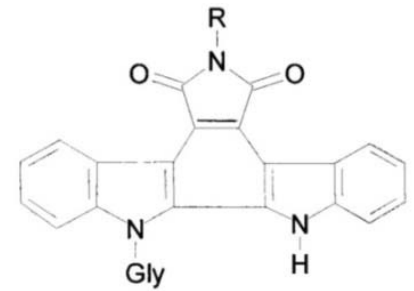

Fig. 1: Generic formula of indolo[2,3-a]pyrollo[3,4-c]carbazole5,7-dione $\mathrm{N}$-glycosides, where Gly stands for pentose residues (L-arabinoses, D-riboses, D-xyloses) and hexoses (D-galactoses) and $R$ is amino group, benzamido group, (pyrid-2-yl)amino group, acetamido group, or urea group

LHS-1269 is $\mathrm{N}-\{12-(\beta-\mathrm{D}$-xylopiranozyl)-5,7-dioxo-indolo[2,3a]pyrollo[3,4-c]carbazole-6-yl\}pyridin-2-carboxamide, an original indolocarbazole derivative with xylose residue. This compound has a pronounced cytotoxic activity $\left(\mathrm{IC}_{50} 1.7 \cdot 10^{-6} \mathrm{M}\right.$ ) in the human colon cancer cell line HCT-116, which is the most susceptible to drugs of this class. The studies in vivo showed a high and long-term antitumor effect on several mice models of continuous solid tumors: Lewis epidermoid lung carcinoma, AKATOL adenocarcinoma of the colon, CC-5 cervical cancer, Ca-755 mammary adenocarcinoma, B-16 melanoma, P-388 ascites lymphocytic leukemia, and Ehrlich ascites carcinoma [5, 6]. Also, LHS-1269 was shown to have an antiangiogenic effect because it can inhibit vasculogenic mimicry of the tumor, i.e., formation of vascular canals by aggressive tumor cells with high-grade cell phenotype, such as melanoma, without endothelial cells and fibroblasts $[7,8]$.

LHS-1269 substance is a yellow amorphous powder that is freely soluble in dimethyl sulfoxide, slightly soluble in acetone and 
benzene, very slightly soluble in ethanol and practically insoluble in water. Low solubility in water presents a challenge for developing the suitable dosage form, leads to inconsistent absorption and low systemic bioavailability. Parenteral administration (intravenous, intramuscular, subcutaneous and other) ensures a considerably high absorption profile and increased bioavailability. However, the low solubility of the drug substance makes intravenous administration of the solutions impossible and dangerous because it might lead to precipitation and vascular obstruction. Various physical, chemical and technological methods are used alone or in combination to increase the solubility and produce an injectable dosage form of a hydrophobic substance [9-11].

Objective of this study was to develop a technology to produce a stable injectable dosage form of LHS-1269.

\section{MATERIALS AND METHODS}

\section{Materials}

LHS-1269 substance (N. N. Blokhin National Medical Research Center of Oncology of the Ministry of Health of Russian Federation), dimethyl sulfoxide (DMSO) (Khimmed, Russia); 95\% ethanol (Flora Kavkaza OSJC, Russia), Kollidon ${ }^{\circledR}$ 17PF (BASF The Chemical Company, Germany), water for injections and purified water. Nylon membrane filters with a diameter of $25 \mathrm{~mm}$ and a pore size of 0.22 um (Pall Eurasia LLC, Russia); polycarbonate membrane filters Nuclepore with a diameter of $25 \mathrm{~mm}$ and a pore size of $0.2 \mathrm{um}$ (Whatman, Great Britain); cellulose ester filter with a diameter of 25 $\mathrm{mm}$ and a pore size of 0.22 um (Merck Millipore, Ireland); polyvinylidene fluoride membrane filters Durapore with a diameter of $25 \mathrm{~mm}$ and a pore size of 0.22 um (Millipore, Ireland); Express Plus $₫$ polyethersulfone membrane filters with a diameter of $25 \mathrm{~mm}$ and a pore size of 0.22 um (Merck Millipore Ltd., Ireland); Stericup ${ }^{\circledR}$ GP Millipore Express $®$ Plus filtration system with polyethersulfone filters with a pore size of 0.22 um (Merck KGaA, Germany).

\section{Equipment}

Laboratory scales DL-120 (AND, Japan), mechanical dispenser Proline Prospenser 1-10 ml (Sartorius, Germany), magnetic stirrer IKA ${ }^{\circ}$ C-MAG HS 4 (IKA-Werke GmbH and Co KG, Germany), overhead mechanical stirrer RZR 2021 Heidolph with propeller stirring element PR 30 Heidolph (Heidolph, Germany), water bath Büchi Heating Bath B-490 (Büchi Labortechnik AG, Switzerland), Lipex $^{\mathrm{TM}}$ extruder (Northern Lipids, Canada), Millipore WP6122050 vacuum pressure pump (Merck, Billerica, MA, USA), water for injection system УВОИ-М-Ф/1812 (MedianaFilter, Russia), freeze dryer Edwards Minifast D0.2 (Ero Electronic SpA, Italy), semiautomatic device ПЗР-34-ВИПС-МЕД for vial capping (VIPSMED LLC, Russia), spectrophotometer Cary 100 (Agilent Technologies, Australia), vibrating viscometer Vibro Viscometer SV10 (AND Company Limited, Japan), pH meter HANNA HI 2211 (Hanna Instruments, Romania).

\section{Methods}

\section{Production of an injectable dosage form of LHS-1269}

The solubilization, dissolution, filtration and freeze-drying steps were performed after preparing model solutions of the studied indolocarbazole derivative.

\section{Quantitative analysis of LHS-1269 in the model solutions}

Assay of the active substance was performed using spectrophotometry with a working reference standard (RS) at $320 \pm 3 \mathrm{~nm}$.

\section{Analysis of pH of LHS-1269 solution}

The $\mathrm{pH}$ value of the samples of LHS-1269 dosage form was determined at 21 to $25{ }^{\circ} \mathrm{C}$. Procedure: Add $8 \mathrm{ml}$ of purified water to the concentrate and mix to obtain a homogenous solution and measure the $\mathrm{pH}$ value.

\section{Analysis of viscosity of LHS-1269 solution}

The dynamic viscosity of the samples of LHS-1269 dosage form was determined at 21 to $25{ }^{\circ} \mathrm{C}$. Procedure: Add $2.9 \mathrm{ml}$ of purified water to the concentrate and mix to obtain a homogenous solution with a 5 $\mathrm{mg} / \mathrm{ml}$ concentration of the active substance. Mix the obtained solutions from 2 vials and measure the viscosity.

\section{Estimation of an average weight of the vial contents}

Ten filled vials with concentrate were sampled. The labels were removed, the vials were weighed individually. The contents were quantitatively removed from each vial, the vials were thoroughly rinsed inside. Each vial was dried and weighed again. The weight of the contents was calculated as the difference between the weight of the filled vial and the empty vial.

\section{RESULTS AND DISCUSSION}

While developing a water-soluble LHS-1269 dosage form, we considered a few pharmaceutical technological approaches that comply with the physical and chemical properties of the active substance. The approaches included solubilization/complexformation, production of solid dispersion, change of the $\mathrm{pH} / \mathrm{salt}$ formation and inclusion into biocompatible phospholipid vesicles, i.e., liposomes. A comparative study of the produced models of the injectable dosage form was conducted. The most effective in vivo and the most technologically acceptable composition included DMSO and ethanol as co-solvents of the hydrophobic substance, solubilizer/complexing agent Kollidon $® 17 \mathrm{PF}$ with $1 / 11 / 32 / 40$ ratio of LHS-1269/DMSO/ethanol/Kollidon $®$ by weight. Concentration of the active substance in the aqueous solution was $5 \mathrm{mg} / \mathrm{ml}$.

Procedure: To prepare an aqueous solution of LHS-1269, add DMSO to the portion of the active substance in $1 / 11$ ratio by weight and mix to obtain a clear red and orange solution. Add a respective volume of ethanol to the solution of LHS-1269 in DMSO. Color of the solution will intensify. Dissolve Kollidon ${ }^{8} 17 \mathrm{PF}$ in water for injection to obtain a clear colorless solution. Heat the mixture to $50-60^{\circ} \mathrm{C}$ on a water bath to speed up the dissolution process. Then add the aqueous solution of Kollidon® portion-wise to the organic solution of LHS-1269. Note that the mixture will become opaque if the LHS-1269 solution is added to the Kollidon ${ }^{\circledR}$ solution. Filter the resulting solution of LHS-1269 through a 0.22 um membrane filter to sterilize it.

Permeability of membranes during filtration of LHS-1269 aqueous solution was evaluated for 5 types of filters: nylon $(\mathrm{N})$, polyethersulfone (PES), polyvinylidene fluoride (PVDF), cellulose ether (CE) and polycarbonate (PC) (table 1).

Table 1: Content of LHS-1269 in the solution depending on the type of filter

\begin{tabular}{|c|c|c|c|c|c|}
\hline \multicolumn{6}{|c|}{ Content of LHS-1269 in the solution, \% } \\
\hline \multirow[t]{2}{*}{ Before filtration } & \multicolumn{5}{|c|}{ After filtration, type of filter } \\
\hline & $\mathbf{N}$ & PES & PVDF & CE & PC \\
\hline 100 & $98.0 \pm 0.53$ & $97.7 \pm 0.50$ & $97.2 \pm 0.69$ & $97.4 \pm 0.51$ & $97.2 \pm 0.42$ \\
\hline
\end{tabular}

Note. The data are given as mean $\pm \mathrm{SD}, \mathrm{n}=3$

The data analysis showed that the content of LHS-1269 in the solution is almost independent of the type of filter. The loss of the active substance was minimal with the use of nylon and polyethersulfone filters. 
The solution of LHS-1269 becomes opaque and forms a precipitate after $3 \mathrm{~d}$ of storage at $+4{ }^{\circ} \mathrm{C}$. Since the model dosage form has low stability, the potential improvement of the stability by freeze drying was considered. Freeze drying of the solution of LHS-1269 was performed by long-term incubation at-45.-47 ${ }^{\circ} \mathrm{C}$ because the solution contains organic solvents with low eutectic points [12]. The sterile aqueous solution of LHS-1269 was dispensed in the portions of $3 \mathrm{ml}$ into $20 \mathrm{ml}$ vials, placed at the shelves of the freeze dryer and frozen at $-47^{\circ} \mathrm{C}$. Then the drug product was incubated for around 12 $\mathrm{h}$ at this temperature. The vacuum pump was turned on afterward. In $3.5 \mathrm{~h}$ after equilibration of the vacuum, the temperature of the shelves was raised to $-45^{\circ} \mathrm{C}$, the drug product was incubated at this temperature for $3.5 \mathrm{~h}$. Them the shelf temperature was raised to- 43 ${ }^{\circ} \mathrm{C}$. At this point, a dome-shaped film appeared at the surface of the frozen mass. When the temperature of the drug product reached $-35{ }^{\circ} \mathrm{C}$, the mass began to break down. As a result, lyophilization was discontinued.

The high content of ethanol probably caused the difficulties with lyophilization of the studied dosage form of LHS-1269. According to the literature [13], alcohol affects the freeze drying of solutions significantly. Duration of the drying is prolonged if the solutions contain $10 \%$ of ethanol. The solutions with $20 \%$ ethanol develop an abnormal shape/structure during freeze-drying, the process is elongated considerably as a result. Various combinations of excipients of different origins (Kollidon $®$ 17PF, Macrogol 400, and Poloxamer 188) were used as solubilizers to decrease ethanol concentration in the studied composition. This decrease, together with the increase of $\mathrm{pH}$ to slightly alkaline value, lowered solubility of LHS-1269 or therapeutic efficacy of the drug.
Freeze drying of the aqueous solution of LHS-1269 is not possible, so we propose producing a stable injectable dosage form of LHS-1269 as a concentrated mixture of drug substance, DMSO, 95\% ethanol and Kollidon $® 17 \mathrm{PF}$. The concentrate is dissolved in water for injection immediately before administration. Production of the dosage form LHS-1269, concentrate for solution for injection and infusion $25 \mathrm{mg}$, includes the following technological stages:

I. Preparation of the solution of LHS-1269. Add the required volume of DMSO to a weighed amount of the active substance, mix with a magnetic stirrer or a propeller stirrer at 200-250 rpm until the complete dissolution of LHS-1269.

II. Preparation of the solution of Kollidon® 17PF. Place a calculated volume of ethanol on a magnetic stirrer in a tightly closed container to prevent evaporation of the solvent. Divide the weighed amount of Kollidon ${ }^{\circledR}$ into approximately 810 equal portions. Add these portions one by one to the liquid during constant stirring with the average rate of magnet stirrer of $4000 \mathrm{rpm}$. Add each subsequent portion of the solubilizer to the solution only after the previous one dissolves. Polyvinylpyrrolidone agglutinates and forms lumps if added excessively, which elongates and complicates the process considerably. A clear alcohol solution of Kollidon ${ }^{\circledR} 17 \mathrm{PF}$ is obtained.

III. Sterilization filtration and dispensing the concentrate. Mix the solutions of LHS-1269 and polyvinylpyrrolidone during constant stirring and filter them with Stericap ${ }^{\circledR}$ system. The sterile concentrate has high viscosity (about $140 \mathrm{mPa} \cdot \mathrm{s}$ ), so it is dispensed into vials by weight. $2.1 \mathrm{~g}$ in a vial, sealed with a rubber seal and aluminum cap. Composition of the concentrate is shown in table 2.

Table 2: Composition of the drug product LHS-1269, concentrate for solution for injection and infusion 25 mg, per vial

\begin{tabular}{lll}
\hline Component of the dosage form & Weigh of the component, $\mathbf{g}$ & Content of the component, \% \\
\hline LHS-1269 & 0.025 & 1.2 \\
DMSO & 0.275 & 13.1 \\
Ethanol 95\% & 0.800 & 38.1 \\
Kollidon® 17PF & 1.000 & 47.6 \\
\hline
\end{tabular}

Three experimental batches of the drug product LHS-1269, concentrate for solution for injection and infusion $25 \mathrm{mg}$, were produced according to the developed technology. Selected quality characteristics of the batches are presented in table 3. Preliminary evaluation of stability of the dosage form showed that shelf-life of the drug product at room temperature is at least $1 \mathrm{y}$. The stability studies are ongoing.

Table 3: Quality characteristics of the experimental batches of LHS-1269, concentrate for solution for injection and infusion $25 \mathrm{mg}$

\begin{tabular}{llll}
\hline Characteristics & Batch & $\mathbf{0 1 0 2 2 0}$ \\
\cline { 2 - 4 } & $\mathbf{0 1 1 1 1 9}$ & $\mathbf{0 1 0 1 2 1}$ \\
\hline Appearance & Viscous clear liquid of a vivid yellow color & $2.08 \pm 0.01$ & \\
Average weight of contents of the vial, $g$ & $2.08 \pm 0.01$ & $3.9 \pm 0.13$ & $2.07 \pm 0.02$ \\
pH of the solution & $3.9 \pm 0.15$ & $25.7 \pm 0.26$ & - \\
Average content of LHS-1269 in the vial, $\mathrm{mg}$ & $25.4 \pm 0.21$ & $8.5 \pm 0.33$ & $23.8 \pm 0.18$ \\
Viscosity* $\mathrm{mPa} \cdot \mathrm{s}$ & $8.4 \pm 0.43$ & $7.8 \pm 0.79$ \\
\hline
\end{tabular}

Note. Viscosity of the $5 \mathrm{mg} / \mathrm{ml}$ solution of LHS-1269 prepared by dissolving the concentrate in water for injection. The data are given as mean \pm SD, $\mathrm{n}=3$.

\section{CONCLUSION}

The study led to development of a technology to produce a stable injectable dosage form of an original hydrophobic derivative of indolocarbazole LHS-1269. The aqueous solution of the studied indolocarbazole derivative has low stability and cannot be lyophilized, so an anhydrous concentrate of LHS-1269 was produced that is dissolved in water for injection immediately before administration. LHS-1269 is a high-potential antitumor drug. Our study showed that it could be formulated in a stable injectable dosage form convenient for clinical practice.

\section{FUNDING}

Nil

\section{AUTHORS CONTRIBUTIONS}

Maria Dmitrieva, Alexander Kolpaksidi: Research design, obtaining and analyzing data, writing the text of the manuscript. Olga Orlova,
Ivan Krasnyuk: Research design, data analysis, verification of the final version of the manuscript. Elena Ignatyeva: Obtaining and analyzing data. Anna Lantsova, Ludmila Nikolaeva: Review of publications, translation.

\section{CONFLICT OF INTERESTS}

The authors declare no conflict of interest.

\section{REFERENCES}

1. Zenkov RG, Ektova LV, Vlasova OA, Belitsky GA, Yakubovskaya MG, Kirsanov KI. Indolo. Carbazoles: diversity, biological properties, application in antitumor therapy. Chem of Heterocyc Comp 2020;56(2):644-58.

2. Kiseleva MP, Pokrovsky VS, Tataskiy VV, Borisova LM, Golubeva IS, Ektova LV. Indolocarbazole derivatives- a promising class of anticancer drugs. Rossijskij bioterapevtičeskij zurnal 2018;17(4):20-6. doi: 10.17650/1726-9784-2018-17-4-20-26. 
3. Apryshko GN, Zhukova OS, Fetisova LV, Vlasenkova NK, Pugacheva $\mathrm{RB}$, Goryunova OV. In silico and in vitro research of potential antineoplastic amino acid derivatives of indolocarbazole glycosides properties. Russ J Biother. 2017;4:46-54.

4. Melnik SYa, Vlasenkova NK, Garaeva LD, Golubeva IS, Goryunova OV, Eremina VA. Method for the preparation of Nglycosides of indolo[2,3-a]pyrrolo[3,4-c]carbazole-5,7-diones with cytotoxic and antitumor activity. Patent No. 2427585 C1. Russian Federation; 2009.

5. Golubeva IS, Yavorskaya NP, Ektova LV, Dmitrieva MV, Borisova LM, Eremina VA. Antitumor activity of some derivatives of indolo[2,3-a]carbazoles N-glycosides with xylose carbohydrate residue. Russ J Biother. 2020;19:86-93 (In Russ.).

6. Golubeva IS, Eremina VA, Moiseeva NI, Ektova LV, Yavorskaya NP. A derivative of the N-glycoside class indolo. Vol. 2. Pyrrolo[3,4-c]carbazole-5,7-diones- $\mathrm{N}-\{\quad 12$-( $\beta$-Dxylopyranosyl)-5,7-dioxo-indolo[2,3-a]pyrrolo[3,4-c] carbazol6-yl\} pyridine-2-carboxamide, with cytotoxic and antitumor activity. Patent No. 2667906 C1 Russian Federation; 2018.

7. Vartanian A, Baryshnikova M, Burova O, Afanasyeva D, Misyurin V, Belyavsky A, Shprakh Z. Inhibitor of vasculogenic mimicry restores sensitivity of resistant melanoma cells to DNA-damaging agents. Melanoma Res. 2017;27(1):8-16. doi: 10.1097/CMR.0000000000000308, PMID 27776018.
8. Vartanyan AA, Baryshnikova MV, Eremina VA, Miniker TD, Tikhonova NI, Kuzmina NE. Indolocarbazole derivatives are blocking vasculogenic mimicry in tumors. Patent No. 2557554 C1. Russian Federation; 2015.

9. Kolluru LP, Atre P, Rizvi SAA. Characterization and applications of colloidal systems as versatile drug delivery carriers for parenteral formulations. Pharmaceut (Basel). 2021;2:108

10. Savjani KT, Gajjar AK, Savjani JK. Drug solubility: importance and enhancement techniques. ISRN Pharm. 2012;2012:195727. doi: $10.5402 / 2012 / 195727$.

11. Sopyan I, Syah ISK, Nurhayti D, Budiman A. Improvement of simvastatin dissolution rate using the derivative noncovalent approach by solvent drop grinding method. Int J App Pharm. 2020;1:21-4. doi: 10.22159/ijap.2020v12i1.35865.

12. Gulyakin I, Lantsova A, Nikolaeva L, Dmitrieva M, Oborotova N, Orlova O, Zhuravleva N. Features of the development of a lyophilized injectable dosage form of the original anticancer drug LCS-1208. Int J App Pharm. 2021;13:102-5. doi: 10.22159/ijap.2021v13i4.41371.

13. Seager H, Taskis CB, Syrop M, Lee TJ. Structure of products prepared by freeze-drying solutions containing organic solvents. J Parenter Sci Technol. 1985;39(4):161-79. PMID 4032158. 